\title{
Conteúdo Corporal e Exigências Líquidas e Dietéticas de Macroelementos Minerais (Ca, P, Mg, Na e K) de Novilhos Mestiços Holandês-Gir em Ganho Compensatório ${ }^{1}$

\author{
Maria Izabel Vieira de Almeida ${ }^{2}$, Carlos Augusto de Alencar Fontes ${ }^{3}$, Fernando Queiroz de \\ Almeida ${ }^{4}$, Oriel Fajardo Campos $^{5}$, Rodrigo Furtado Guimarães ${ }^{2}$
}

\begin{abstract}
RESUMO - Foram utilizados 39 novilhos mestiços Holandês-Gir, com o objetivo de avaliar a influência do ganho compensatório sobre o conteúdo corporal e as exigências líquidas e dietéticas de cálcio, fósforo, magnésio, sódio e potássio de bovinos. Trinta animais foram submetidos à alta pressão de pastejo, enquanto nove tiveram oferta ilimitada de pasto (grupo ganho contínuo), durante 114 dias, após o qual seis animais do primeiro grupo e três do segundo grupo foram abatidos e os restantes, confinados. Entre os animais do primeiro grupo, 12 receberam alimentação ad libitum (grupo ganho compensatório) e 12 receberam alimento 15\% acima da mantença (grupo mantença), enquanto os seis animais do grupo ganho contínuo receberam a mesma dieta ad libitum. Foram ajustadas equações de regressão do logaritmo do conteúdo corporal dos minerais, em função do logaritmo do peso corporal vazio (PCVZ). As exigências de minerais não diferiram quanto ao tratamento, e as exigências líquidas para ganho de $1 \mathrm{~kg}$ PCVZ variaram de 10,04 a 12,38 g para cálcio, 9,33 a 9,41 g para fósforo, 0,50 a 0,38 g para magnésio, 1,22 a 1,07 g para sódio e 2,24 a 2,75 g para potássio, para novilhos com pesos entre $150 \mathrm{e}$ $450 \mathrm{~kg}$, respectivamente. As exigências dietéticas, para ganho de $1 \mathrm{~kg}$ de peso vivo, variaram de 15,88 a 25,68 g para cálcio, 16,62 a $10,35 \mathrm{~g}$ para fósforo, 5,12 a 9,82 g para magnésio, 2,25 a 4,35 g para sódio e 18,23 a 49,18 g para potássio.
\end{abstract}

Palavras-chave: bovino, conteúdo corporal, macrominerais, requerimentos nutricionais

\section{Body Content and Net and Dietary Requirements of Macrominerals (Ca, P, Mg, Na and K) of Crossbred Holstein-Gyr Steers during Compensatory Growth}

\begin{abstract}
Thirty-nine crossbreed Holstein-Gyr steers were used aiming to evaluate the influence of compensatory growth on body content and on net and dietary requirements of calcium, phosphorus, magnesium, sodium, and potassium of bovines. Thirty animals were submitted to a high pasture stocking rate, while nine had free access to the pasture (continuous growth group), during 114 days. After that, six animals from the first group and three from the second group were slaughtered, and the remaining animals were feedlot confined. From the animals of the first group, twelve animals were full fed (compensatory growth group) and twelve animals were fed $15 \%$ above the maintenance level (maintenance group), while the six animals from continuous gain group were full fed the same diet. Regression equations of $\log \mathrm{kg}$ body contents of each mineral as a function of $\log$ empty-body weight (EBW) were fitted. The macrominerals requirements did not differ regarding to the treatments, and the net requirements for $1 \mathrm{~kg}$ of EBW gain ranged from 10.04 to $12.38 \mathrm{~g}$ for calcium; 9.33 to 9.41 $\mathrm{g}$ for phosphorus; 0.50 to $0.38 \mathrm{~g}$ for magnesium; 1.22 to $1.07 \mathrm{~g}$ for sodium; and 2.24 to $2.75 \mathrm{~g}$ for potassium, for steers with weights between 150 to $450 \mathrm{~kg}$, respectively. The dietetic macrominerals requirements, for $1 \mathrm{~kg}$ of liveweight of gain, ranged from $15.88 \mathrm{to} 25.68 \mathrm{~g}$ for calcium; 16.62 to $10.35 \mathrm{~g}$ for phosphorus; 5.12 to $9.82 \mathrm{~g}$ for magnesium; 2.25 to $4.35 \mathrm{~g}$ for sodium; and 18.23 to 49.18 for potassium.
\end{abstract}

Key Words: bovine, body content, macrominerals, nutritional requirements

\section{Introdução}

A concentração corporal de minerais não é constante, uma vez que depende das proporções dos tecidos ósseo, muscular e adiposo, os quais não aumentam na mesma proporção durante o crescimento. Assim, as exigências são influenciadas, principalmente, pela condição fisiológica (AMMERMAN e GOODRICH, 1983; ROBELIN e GEAY, 1984), pelo sexo (GRACE, 1983) e pela raça (NOUR e THONEY, 1988).

Desprezando as variações nas concentrações desses elementos com o aumento no peso de corpo vazio (PCVZ) de animais entre 75 e $500 \mathrm{~kg}$, o AGRICULTURAL RESEARCH COUNCIL - ARC (1980) preconizou valores constantes de requerimentos de 14,0 e 8,0 g/kg PV ou PCVZ ganho, para Ca e $\mathrm{P}$, respectivamente. O AGRICULTURAL AND

\footnotetext{
${ }^{1}$ Parte da tese apresentada à Universidade Federal de Viçosa para obtenção do título de Doctor Scientiae.

2 UFV/DZO - 36571-000 - Viçosa, MG. E.mail: miva@homenet.com.br

3 UENF/CCTA - 28015-820 - Campos dos Goytacazes, RJ. E.mail: caafontes@uenf.br

4 UFRRJ/DMCV-IV - 23851-970 - Seropédica, RJ. E.mail: falmeida@ufrrj.br

5 EMBRAPA/CNPGL - 36.038-330 - Juiz de Fora, MG. E.mail: oriel@cnpgl.embrapa.br
} 
FOOD RESEARCH COUNCIL - AFRC (1991) adotou equações baseadas no crescimento ósseo para estimar as exigências de $\mathrm{Ca}$ e $\mathrm{P}$, considerando que a deposição desses elementos no corpo é reduzida com o aumento de gordura corporal, uma vez que esta praticamente não contém Ca e os triglicerídeos depositados no tecido adiposo contêm pequenas quantidades de P. Para bovinos de 300, 400 e $500 \mathrm{~kg}$ de $\mathrm{PV}$, os requerimentos líquidos estimados de $\mathrm{Ca}$, por $\mathrm{kg}$ de ganho de PV seriam de 12,7, 11,9 e 11,6 g, respectivamente, e para $\mathrm{P}$ os requerimentos líquidos de animais de mesmos pesos vivos, de 7,2, 6,8 e $6,7 \mathrm{~g} / \mathrm{kg}$ PV. respectivamente, O NATIONAL RESEARCH COUNCIL (1984, 1996) estimou os requerimentos líquidos de cálcio e fósforo para ganho de peso de acordo com o peso vivo e o ganho de proteína.

No Brasil, diversos pesquisadores têm estudado a composição corporal e as exigências de minerais de bovinos (LANA et al., 1992; PIRES et al., 1993a,b; CASTRO et al., 1993; SOARES, 1994; CARVALHO, 1989; FERREIRA et al., 1999; ARAÚJO et al., 1998; SIGNORETTI et al., 1999; PAULINO et al., 1999). Entretanto, os resultados obtidos são ainda insuficientes para publicação de uma tabela de requerimentos adaptada às condições locais. Além disto, admite-se que o ganho compensatório seja fenômeno inerente ao sistema predominante de produção de bovinos de corte no país e que sua influência sobre os requerimentos de minerais não foi ainda estabelecida. Portanto, este trabalho objetivou determinar o conteúdo corporal de macroelementos minerais $(\mathrm{Ca}, \mathrm{P}, \mathrm{Mg}, \mathrm{K}$ e $\mathrm{Na}$ ) e estimar as exigências líquidas e dietéticas desses minerais para novilhos mestiços Holandês-Gir, em fase de ganho de peso compensatório.

\section{Material e Métodos}

Foram comparados novilhos mestiços HolandêsGir, confinados, com peso vivo médio de $202,1 \pm 49,1 \mathrm{~kg}$ e idade de 19,3 $\pm 5,1$ meses, que haviam sido submetidos à restrição de pasto, durante 104 dias após a estação seca, de forma a manterem o peso corporal constante (grupo de ganho compensatório), com novilhos semelhantes, que, após a seca, tiveram oferta ilimitada de pasto no período de 104 dias que antecederam ao confinamento (grupo de ganho contínuo).

O local de realização do experimento, a dieta utilizada, os animais, os procedimentos de adaptação dos animais à dieta e às instalações, as condições de manejo, bem como os procedimentos de abate, coleta de amostras, análises químicas e estatísticas, foram descritos com detalhes por ALMEIDA et al. (2001).

A solução mineral para determinação da concentração dos macroelementos minerais foi preparada por via úmida. A determinação do fósforo foi feita por espectrofotometria (colorimetria), enquanto o cálcio e o magnésio foram determinados por espectrofotometria de absorção atômica e o sódio e o potássio, por espectrofotometria de chama, segundo SILVA (1990).

A determinação de matéria seca (MS), matéria orgânica (MO), fibra em detergente neutro (FDN), extrato etéreo (EE) e energia bruta (EB) dos alimentos, das sobras e das fezes foi feita conforme descrito por SILVA (1990) (Tabela 1). As análises de nitrogênio total foram realizadas em aparelho semimicro Kjeldahl, com exceção das análises de silagem de milho, que foram realizadas em aparelho macro Kjeldahl.

Os conteúdos corporais de macroelementos minerais foram determinados por meio das análises das amostras de todos os componentes corporais, incluindo vísceras, órgãos, couro, sangue, cauda, cabeça, pés, músculos, tecido adiposo e ossos, estabelecendo-se médias ponderadas, considerando o peso relativo de cada parte do corpo.

As porcentagens de músculos, tecido adiposo e ossos da carcaça foram estimadas a partir de suas proporções na secção transversal compreendida entre a 9a e a 11a costela (secção HH) (HANKINS e HOWE, 1946), retirada da meia-carcaça esquerda, utilizando as equações propostas pelos autores:

Músculo: $\hat{Y}=16,08+0,80 X$

Tecido adiposo: $\hat{Y}=3,54+0,80 X$

Ossos: $\hat{Y}=5,52+0,57 X$

em que $X$ é a porcentagem dos componentes na secção HH.

O peso corporal vazio (PCVZ) dos animais foi determinado por meio da soma do peso da carcaça, do sangue, da cabeça, dos pés, do couro, da cauda, dos órgãos e das vísceras. A relação entre o PCVZ e o peso vivo (PV) dos animais-referência, representativos dos animais experimentais e abatidos ao início do experimento, foi determinada, e o valor obtido foi usado na estimativa do PCVZ inicial dos animais remanescentes. O PCVZ final dos animais experimentais foi determinado após o abate, somando-se os pesos das partes do corpo, de modo semelhante ao obtido para os animais-referência. 
Rev. bras. zootec.

Tabela 1 - Teores médios de matéria seca $(M S)$, proteína bruta $(\mathrm{PB})$, extrato etéreo (EE), energia bruta (EB), energia metabolizável $(E M)$, cálcio $(\mathrm{Ca})$, fósforo $(\mathrm{P})$, magnésio $(\mathrm{Mg})$, sódio $(\mathrm{Na})$ e potássio $(\mathrm{K})$ da ração

Table 1 - Average contents of dry matter (DM), crude protein (CP), ether extract (EE), gross energy (CE), metabolizable energy (ME), calcium $(\mathrm{Ca})$, phosphorus $(\mathrm{P})$, magnesium $(\mathrm{Mg})$, sodium $(\mathrm{Na})$ and potassium $(K)$ in the diet

\begin{tabular}{|c|c|c|c|c|c|c|c|c|c|c|c|}
\hline \multirow[t]{2}{*}{$\begin{array}{l}\text { Ingrediente } \\
\text { Ingredient }\end{array}$} & \multirow[t]{2}{*}{$\begin{array}{l}\text { (\%) na dieta } \\
\text { (\% of diet) }\end{array}$} & \multirow{2}{*}{$\begin{array}{l}\text { MS } \\
D M \\
(\%)\end{array}$} & \multicolumn{7}{|c|}{$\begin{array}{c}\text { Composição da dieta (\%MS) } \\
\text { Diet composition }(\% D M)\end{array}$} & \multirow[b]{2}{*}{$\begin{array}{l}\mathrm{Na} \\
(\%)\end{array}$} & \multirow[b]{2}{*}{$\underset{(\%)}{\mathrm{K}}$} \\
\hline & & & $\begin{array}{l}\mathrm{PB} \\
C P\end{array}$ & $\begin{array}{l}\mathrm{EE} \\
E E\end{array}$ & $\begin{array}{l}\mathrm{EB} \\
C E \\
(\mathrm{Mc}\end{array}$ & $\begin{array}{c}\text { EM } \\
M E \\
1 / \mathrm{kg})\end{array}$ & $\begin{array}{l}\mathrm{Ca} \\
(\%)\end{array}$ & $\begin{array}{l}\mathrm{P} \\
(\%)\end{array}$ & $\begin{array}{l}\mathrm{Mg} \\
(\%)\end{array}$ & & \\
\hline $\begin{array}{l}\text { Silagem de milho } \\
\text { Corn silage }\end{array}$ & 73,97 & 24,07 & 9,5 & 2,7 & 3,90 & - & 0,73 & 0,59 & 0,15 & 0,15 & 1,46 \\
\hline $\begin{array}{l}\text { Concentrado + mistura mineral }{ }^{1} \\
\text { Concentrate }+ \text { mineral mixture }\end{array}$ & 26,03 & 88,43 & 27,0 & 2,9 & 3,74 & - & 0,81 & 0,50 & 0,16 & 0,18 & 1,57 \\
\hline $\begin{array}{l}\text { Dieta } \\
\text { Diet }\end{array}$ & 100 & 40,82 & 14,1 & 2,8 & 3,86 & 2,34 & 0,75 & 0,57 & 0,15 & 0,16 & 1,49 \\
\hline
\end{tabular}

${ }^{1}$ Mistura mineral: $1,95 \%$ da dieta.

${ }^{1}$ Mineral mixture: $1.95 \%$ in diet.

Para descrever as alterações dos conteúdos corporais de $\mathrm{Ca}, \mathrm{P}, \mathrm{Mg}$, $\mathrm{Na}$ e $\mathrm{K}$, retidos no corpo animal com a elevação do peso corporal, foram ajustadas equações de regressão do logaritmo dos pesos $(\mathrm{kg})$ de cada mineral, em função do logaritmo do PCVZ (ARC, 1980), segundo o modelo:

$$
Y_{i j}=\mu+b_{i} X_{i j}+e_{i j}
$$

em que $\mathrm{Y}_{\mathrm{ij}}=$ logaritmo do conteúdo total de $\mathrm{Ca}, \mathrm{P}$, $\mathrm{Mg}, \mathrm{Na}$ ou K ( $\mathrm{kg}$ ) retido no corpo vazio, do animal $\mathrm{j}$, do tratamento $\mathrm{i} ; \mu=$ efeito de média (intercepto); $\mathrm{b}_{\mathrm{i}}=$ coeficiente de regressão do logaritmo do conteúdo de $\mathrm{Ca}, \mathrm{P}, \mathrm{Mg}, \mathrm{Na}$ e K, em função do logaritmo do PCVZ; Xij = logaritmo do PCVZ do animal j do tratamento $i$, em que $i=1$, ganho contínuo e $2=$ ganho compensatório; e $\mathrm{e}_{\mathrm{ij}}=$ erro aleatório, associado a cada observação, pressuposto normalmente distribuído, com média zero e variância $\sigma^{2}$.

As exigências líquidas dos macroelementos inorgânicos para ganho de $1 \mathrm{~kg}$ de PCVZ foram estabelecidas a partir de equações obtidas por derivação das equações de regressão anteriormente referidas, obtendo-se equações do tipo:

$$
\mathrm{Y}=\mathrm{b} \cdot 10^{\mathrm{a}} \cdot \mathrm{PCVZ}^{(\mathrm{b}-1)}
$$

em que $\mathrm{Y}=$ exigência líquida do mineral; $\mathrm{a}=$ intercepto; $\mathrm{e} \mathrm{b}=$ coeficiente de regressão das equações de predição dos conteúdos corporais de macroelementos minerais.

Os requerimentos dietéticos dos macroelementos inorgânicos foram estimados pelo método fatorial, como proposto pelo ARC (1980), para animais que pesavam entre $150 \mathrm{e} 450 \mathrm{~kg}$, ganhando $1 \mathrm{~kg}$ de $\mathrm{PV}$ por dia, com base nas seguintes equações:

$$
\begin{gathered}
\mathrm{RL}=\mathrm{G}+\mathrm{E} \\
\mathrm{RD}=(\mathrm{RL} / \mathrm{D}) * 100
\end{gathered}
$$

em que $\mathrm{RL}=$ requerimento líquido total; $\mathrm{G}=$ retenção diária do elemento mineral; $\mathrm{E}=$ perda endógena do elemento nas fezes e na urina; $\mathrm{RD}=$ requerimento dietético; $\mathrm{D}=$ disponibilidade do elemento na dieta.

Os valores de perdas endógenas e de disponibilidades de $\mathrm{Ca}, \mathrm{P}, \mathrm{Mg}$, Na e $\mathrm{K}$ utilizados para cálculo dos requerimentos dietéticos estão relacionados na Tabela 2.

A conversão do peso corporal vazio para peso vivo, no intervalo de pesos estudado, foi feita pela regressão do $\mathrm{PCVZ}$ dos animais utilizados no experimento, de acordo com seus PV. Na conversão das exigências de minerais para ganho de $1 \mathrm{~kg}$ de peso corporal vazio em exigência para ganho de $1 \mathrm{~kg}$ de peso vivo, foi utilizado o fator médio de 1,19 , obtido no presente estudo.

No ajustamento das equações de regressão, foi utilizado o programa LSMLMW (HARVEY, 1987). A identidade das equações obtidas para os tratamentos ganho contínuo e ganho compensatório foi avaliada, utilizando-se o teste de identidade de modelos (GRAYBILL, 1976). Na análise de variância, utilizou-se o teste de $\mathrm{F}$, a $5 \%$ de probabilidade. 
ALMEIDA et al.

Tabela 2 - Perdas endógenas totais e biodisponibilidades de cálcio, fósforo, magnésio, sódio e potássio ${ }^{1}$ Table 2 - Total endogenous losses and bioavailabilities of calcium, phosphorus, magnesium, sodium and potassium ${ }^{1}$

\begin{tabular}{|c|c|c|}
\hline $\begin{array}{l}\text { Elemento } \\
\text { Element }\end{array}$ & $\begin{array}{l}\text { Perda endógena total } \\
\text { Total endogenous losses }\end{array}$ & $\begin{array}{c}\text { Disponibilidade }(\%) \\
\text { Bioavailability }\end{array}$ \\
\hline $\begin{array}{l}\text { Cálcio } \\
\text { Calcium }^{2}\end{array}$ & {$[-0,74+0,0079 \mathrm{PV}(L W)+0,66 * \mathrm{CMS}(D M I)]$} & 68 \\
\hline $\begin{array}{l}\text { Fósforo } \\
\text { Phosphorus } 2\end{array}$ & $1,6 *[-0,06+0,693 * \mathrm{CMS}(D M I)]$ & 58 \\
\hline $\begin{array}{l}\text { Magnésio } \\
\text { Magnesium }\end{array}$ & $3,0 \mathrm{mg} / \mathrm{kg} \mathrm{PV} / \mathrm{dia}$ (LW/day) & 17 \\
\hline $\begin{array}{l}\text { Sódio } \\
\text { Sodium } \\
\text { Potássio }\end{array}$ & $6,8 \mathrm{mg} / \mathrm{kg} \mathrm{PV} / \mathrm{dia}$ (LW/day) & 91 \\
\hline $\begin{array}{l}\text { Potassium } \\
\text { Fecal }\end{array}$ & $2,6 \mathrm{~g} / \mathrm{kg} \mathrm{MS}$ consumida ${ }^{2}$ (Ingested DM) & 100 \\
\hline $\begin{array}{l}\text { Fecal } \\
\text { Urinária }\end{array}$ & $37,5 \mathrm{mg} / \mathrm{kg} \mathrm{PV} / \mathrm{dia}(L W /$ day $)$ & \\
\hline $\begin{array}{l}\text { Urinary } \\
\text { Salivar }\end{array}$ & $0,7 \mathrm{~g} / 10 \mathrm{~kg} \mathrm{PV} / \mathrm{dia}(L W / d a y)$ & \\
\hline $\begin{array}{l}\text { Salivary } \\
\text { Epidérmica } \\
\text { Epidermical }\end{array}$ & $1,1 \mathrm{~g} / \mathrm{dia}($ day) & \\
\hline
\end{tabular}

1 Valores obtidos do ARC (1980) e do AFRC (1991).

2 Considerando consumo de matéria seca (CMS) de $2,4 \%$ do PV.

1 Data from ARC (1980) and from AFRC (1991).

2 Considering dry matter intake (DMI) of $2.4 \%$ LW.

\section{Resultados e Discussão}

Os parâmetros das equações de regressão do logaritmo dos conteúdos de cálcio, fósforo, magnésio, sódio e potássio no corpo vazio, em função do logaritmo do PCVZ, podem ser observados na Tabela 3. Equação única, comum aos animais dos tratamentos ganho contínuo e ganho compensatório, foi adotada para cada um dos elementos minerais, uma vez que o teste de identidade de modelos não revelou diferença $(P>0,05)$ entre os tratamentos, com relação aos minerais. Os animais do ganho contínuo haviam experimentado ganhos apenas moderados nas fases anteriores ao confinamento. Com a melhoria do nível nutricional, mesmo os animais em confinamento exibiram grande aumento no ritmo de crescimento corporal, tendo, aparentemente, crescimento esquelético similar ao dos animais do tratamento ganho compensatório.

Os coeficientes de determinação $\left(\mathrm{r}^{2}\right)$ encontrados para as equações de regressão mostraram-se elevados, estando próximos aos valores encontrados por ARAÚJO et al. (1998) e FERREIRA et al. (1999), e superiores aos obtidos por CARVALHO (1989), LANA et al. (1992), PIRES et al. (1993a,b), SOARES (1994), SIGNORETTI et al. (1999) e PAULINO et al. (1999). A partir dessas equações, foi possível estimar os conteúdos corporais totais e por unidade de PCVZ de cálcio, fósforo, magnésio, sódio e potássio no corpo vazio, para animais de 150 a $450 \mathrm{~kg}$ de PV ou 116 a $399 \mathrm{~kg}$ de PCVZ (Tabelas 4 e 5).

Os resultados dos conteúdos de macroelementos minerais no corpo dos animais mostraram que houve acréscimo de todos eles com o aumento do PCVZ, quando este variou de 116 a $399 \mathrm{~kg}$. O aumento dos conteúdos de $\mathrm{Ca}, \mathrm{P}$ e $\mathrm{Mg}$ estaria relacionado com o crescimento do esqueleto e, especialmente, com a maior calcificação (mineralização) dos ossos.

Tabela 3 - Parâmetros das equações de regressão do logaritmo do conteúdo de $\mathrm{Ca}, \mathrm{P}, \mathrm{Mg}, \mathrm{Na}$ e $\mathrm{K}$ no corpo vazio, em função do logaritmo do PCVZ, para novilhos mestiços dos tratamentos ganho contínuo e ganho compensatório

Table 3 - Parameters of regression equations for logarithm of empty body content of $\mathrm{Ca}, \mathrm{P}, \mathrm{Mg}, \mathrm{Na}$ and $\mathrm{K}$, in function of logarithm of EBW, for crossbred steers in continuous and compensatory gain groups

\begin{tabular}{lccc}
\hline $\begin{array}{l}\text { Mineral } \\
\text { Mineral }\end{array}$ & $\begin{array}{c}\text { Intercepto } \\
\text { Intercept }\end{array}$ & $\begin{array}{c}\text { Coeficiente } \\
\text { Coefficient }\end{array}$ & $\mathrm{r}^{2}$ \\
\hline $\mathrm{Ca}$ & $-2,416068$ & 1,1694755 & 0,875 \\
$\mathrm{P}$ & $-2,044659$ & 1,0059998 & 0,827 \\
$\mathrm{Mg}$ & $-2,702565$ & 0,7672527 & 0,878 \\
$\mathrm{Na}$ & $-2,634753$ & 0,8898203 & 0,944 \\
$\mathrm{~K}$ & $-3,054313$ & 1,164306 & 0,812 \\
\hline
\end{tabular}


Rev. bras. zootec.

Tabela 4 - Estimativas do conteúdo corporal de $\mathrm{Ca}, \mathrm{P}, \mathrm{Mg}$, $\mathrm{Na} \mathrm{e} \mathrm{K,} \mathrm{em} \mathrm{kg,} \mathrm{no} \mathrm{peso} \mathrm{de} \mathrm{corpo} \mathrm{vazio} \mathrm{(PCVZ)} \mathrm{para}$ novilhos mestiços dos tratamentos ganho contínuo e ganho compensatório

Table 4 - Estimated empty body weight (EBW) contents of $\mathrm{Ca}, P$, $\mathrm{Mg}, \mathrm{Na}$ and $\mathrm{K}$, in $\mathrm{kg}$, for crossbred steers in continuous gain and compensatory gain groups

\begin{tabular}{|c|c|c|c|c|c|c|}
\hline \multirow{2}{*}{$\begin{array}{l}\text { PV } \\
L W \\
(\mathrm{~kg})\end{array}$} & \multirow{2}{*}{$\begin{array}{c}\text { PCVZ } \\
E B W \\
(\mathrm{~kg})\end{array}$} & \multicolumn{5}{|c|}{$\begin{array}{l}\text { Conteúdo corporal de minerais }(\mathrm{kg}) \\
\text { Minerals body content }\end{array}$} \\
\hline & & $\mathrm{Ca}$ & $\mathrm{P}$ & $\mathrm{Mg}$ & $\mathrm{Na}$ & $\mathrm{K}$ \\
\hline 150 & 116 & 1,00 & 1,08 & 0,08 & 0,16 & 0,22 \\
\hline 200 & 163 & 1,48 & 1,52 & 0,10 & 0,22 & 0,33 \\
\hline 250 & 210 & 2,00 & 1,96 & 0,12 & 0,27 & 0,45 \\
\hline 300 & 257 & 2,53 & 2,40 & 0,14 & 0,32 & 0,57 \\
\hline 350 & 304 & 3,08 & 2,84 & 0,16 & 0,38 & 0,69 \\
\hline 400 & 352 & 3,64 & 3,29 & 0,18 & 0,43 & 0,81 \\
\hline 450 & 399 & 4,22 & 3,73 & 0,20 & 0,48 & 0,94 \\
\hline
\end{tabular}

$\mathrm{O}$ aumento verificado na concentração de Ca no corpo vazio, com o incremento no peso de corpo vazio dos animais, está de acordo com os resultados obtidos por ARAÚJO et al. (1998), para animais com o mesmo tipo racial, e por SIGNORETTI et al. (1999), para bezerros inteiros da raça Holandesa. Estes comportamentos diferem dos relatados por COELHO DA SILVA (1995), FONTES (1995), FERREIRA et al. (1999), PAULINO et al. (1999) e AFRC (1991). Tal fato pode estar relacionado ao estádio de desenvolvimento dos animais utilizados, pois, no presente experimento e nos descritos por ARAÚJO et al. (1998) e SIGNORETTI et al. (1999), os animais encontravam-se em fase de crescimento, portanto, a deposição de ossos e músculos supera a deposição de gordura, enquanto nas demais citações os animais utilizados estavam em fase de terminação, quando pode haver efeito de diluição, devido ao aumento da gordura corporal, que possui baixo teor de minerais (ARC, 1980).

Embora as equações de regressão, ao apontarem tendência de elevação das concentrações de $\mathrm{Ca}$ e P por unidade de PCVZ, com aumento do peso corporal, estejam em discordância com os resultados de FONTES (1995), os conteúdos médios dos citados minerais no peso ganho encontram-se dentro do intervalo verificado por este e outros autores, no Brasil. Assim, o conteúdo de Ca no PCVZ, para animais de $300 \mathrm{~kg}$ de PV, foi estimado em 9,83 g, valor inferior aos observados por PIRES et al. (1993a), de 22,29 g/kg PCVZ, e por LANA et al. (1992), de 18,10 g/kg PCVZ, para animais de raças de corte, por SOARES (1994), de 21,78 e 17,97 g/kg
853

Tabela 5 - Estimativas do conteúdo corporal de $\mathrm{Ca}, \mathrm{P}, \mathrm{Mg}$, $\mathrm{Na}$ e K, em $\mathrm{g}$ por $\mathrm{kg}$ de peso corporal vazio (PCVZ), de novilhos mestiços dos tratamentos ganho contínuo e ganho compensatório

Table 5 - Estimated empty body weight contents of $\mathrm{Ca}, \mathrm{P}, \mathrm{Mg}, \mathrm{Na}$ and $K$, in $g$ per $\mathrm{kg}$ of empty body weight (EBW), for crossbred steers in continuous gain and compensatory gain groups

\begin{tabular}{lcccccc}
\hline PV & PCVZ & \multicolumn{5}{c}{ Conteúdo corporal de minerais (kg) } \\
$L W$ & $E B W$ & \multicolumn{5}{c}{ Minerals body content } \\
\cline { 3 - 7 }$(\mathrm{kg})$ & $(\mathrm{kg})$ & $\mathrm{Ca}$ & $\mathrm{P}$ & $\mathrm{Mg}$ & $\mathrm{Na}$ & $\mathrm{K}$ \\
\hline 150 & 116 & 8,59 & 9,28 & 0,66 & 1,37 & 1,93 \\
200 & 163 & 9,10 & 9,30 & 0,61 & 1,32 & 2,04 \\
250 & 210 & 9,50 & 9,32 & 0,57 & 1,29 & 2,13 \\
300 & 257 & 9,83 & 9,33 & 0,55 & 1,26 & 2,20 \\
350 & 304 & 10,11 & 9,34 & 0,52 & 1,23 & 2,26 \\
400 & 352 & 10,36 & 9,35 & 0,51 & 1,22 & 2,31 \\
450 & 399 & 10,58 & 9,35 & 0,49 & 1,20 & 2,36 \\
\hline
\end{tabular}

PCVZ, para bovinos mestiços e búfalos e para animais da raça Nelore, respectivamente, e por PAULINO et al. (1999), de 20,78 g/kg PCVZ, para animais de quatro raças zebuínas. Em animais de $350 \mathrm{~kg}$ de PV, o conteúdo estimado de Ca no PCVZ foi de 10,11 g, inferior ao observado por FERREIRA et al. (1999), de 15,59 g/kg PCVZ, para novilhos F1 Simental-Nelore.

De modo semelhante ao verificado para $\mathrm{Ca}$, a concentração de $\mathrm{P}$ no peso de corpo vazio dos animais aumentou ligeiramente com o incremento de peso dos animais, ao contrário do observado por LANA et al. (1992), PIRES et al. (1993a), SOARES (1994), ARAÚJO et al. (1998), FERREIRA et al. (1999), PAULINO et al. (1999) e SIGNORETTI et al. (1999). O conteúdo de P no PCVZ para animais com $300 \mathrm{~kg}$ de PV foi estimado em 9,33 g, valor próximo aos observados por LANA et al. (1992), SOARES (1994) e PAULINO et al. (1999), que foram de 11,02, 11,04 e 9,60 g, respectivamente, sendo superior ao valor proposto pelo ARC (1980), de 8,00 g, e inferior ao observado por PIRES (1993a), de $14,72 \mathrm{~g}$. Em animais de $350 \mathrm{~kg}$ de PV, o conteúdo estimado de P no PCVZ foi de 9,34 g, similar ao observado por FERREIRA et al. (1999), de 9,90 g/kg PCVZ, para novilhos F1 Simental-Nelore.

De modo geral, as concentrações de $\mathrm{Ca}$ e $\mathrm{P}$ encontram-se dentro do intervalo estabelecido pelos trabalhos realizados no Brasil. A tendência verificada no presente trabalho de elevação do conteúdo dos citados elementos por unidade de peso, embora possa ter sido influenciada pela amostra de animais utilizada no experimento, evidencia que durante a fase de 
ganho compensatório ocorre mineralização mais rápida dos ossos, que pode ter sido prejudicada durante o período de estresse nutricional.

A taxa de deposição de $\mathrm{Mg}$ decresceu com o aumento no peso de corpo vazio, não acompanhando a tendência verificada para Ca e P. Este comportamento está de acordo com o observado por diversos autores (LANA et al., 1992; PIRES et al., 1993b; SOARES, 1994; FERREIRA et al., 1999 e PAULINO et al., 1999), enquanto o ARC (1980) propõe uma composição constante de $0,45 \mathrm{~g} / \mathrm{kg}$ PCVZ.

O conteúdo de $\mathrm{Mg}$ encontrado para animais com $300 \mathrm{~kg}$ de PV foi de $0,55 \mathrm{~g} / \mathrm{kg}$ PCVZ, valor próximo aos valores obtidos por LANA et al. (1992), PIRES et al. (1993b) e SOARES (1994), de 0,50, 0,498 e $0,466, \mathrm{~g} / \mathrm{kg}$ PCVZ, respectivamente, e superior ao valor obtido por PAULINO et al. (1999), de 0,356 g/kg PCVZ. Em animais de $350 \mathrm{~kg}$ de PV, o conteúdo estimado de Mg no PCVZ foi de 0,52 g, superior ao observado por FERREIRA et al. (1999), de 0,41 g/kg PCVZ, para novilhos F1 Simental-Nelore.

Semelhante ao observado para o $\mathrm{Mg}$, a concentração de $\mathrm{Na}$ no corpo vazio decresceu com o aumento do PCVZ. O conteúdo de $\mathrm{Na}$, em g/kg de PCVZ, para animais com $300 \mathrm{~kg}$ de PV, foi de $1,26 \mathrm{~g} / \mathrm{kg}$ PCVZ, valor próximo aos obtidos por LANA et al. (1992) e PIRES et al. (1993b), de 1,23 e $1,30 \mathrm{~g} / \mathrm{kg}$ PCVZ, respectivamente. e inferior aos valores obtidos por SOARES (1994) e PAULINO et al. (1999), de 1,63 e 1,49 g/kg PCVZ, respectivamente. Em animais de $350 \mathrm{~kg}$ de PV, o conteúdo estimado de Na no PCVZ foi de 1,23 g, inferior ao observado por FERREIRA et al. (1999), de 1,95 g/kg PCVZ, para novilhos F1 Simental-Nelore.

A concentração de $\mathrm{K}$ no corpo vazio aumentou com o peso, devendo-se ressaltar que, para animais com peso de $300 \mathrm{~kg}$ de PV, obteve-se o valor de 2,20 g/kg PCVZ, próximo aos valores citados por ARC (1980), LANA et al. (1992), PIRES et al. (1993b), SOARES (1994) e PAULINO et al. (1999), de 2,00, 1,77, 2,11, 2,43 e 2,29 g/kg PCVZ, respectivamente. Assim como para animais de $350 \mathrm{~kg}$ de PV, o conteúdo estimado de K no PCVZ foi de 2,26 g, próximo ao observado por FERREIRA et al. (1999), de 2,00 g/kg PCVZ, para novilhos F1 Simental-Nelore.

As exigências líquidas de macroelementos minerais, em gramas por quilograma de ganho de peso de corpo vazio, para ganho de peso de animais com peso vivo entre 150 e $450 \mathrm{~kg}$, estão mostradas na Tabela 6.

A exigência líquida de $\mathrm{Ca}$, para animais com $300 \mathrm{~kg}$ de peso vivo, foi estimada em $11,49 \mathrm{~g}$ por $\mathrm{kg}$
Tabela 6 - Exigências líquidas diárias de macroelementos minerais para ganhos de $1 \mathrm{~kg}$ de peso corporal vazio (GPCVZ) de novilhos mestiços dos tratamentos ganho contínuo e ganho compensatório

Table 6 - Estimated net requirements of macrominerals for gain of $1 \mathrm{~kg}$ of empty body weight (EBWG), for crossbred steers in continuous gain and compensatory gain groups

\begin{tabular}{lcccccc}
\hline PV & PCVZ & \multicolumn{6}{c}{ Exigências líquidas diárias (g/kg GPCVZ) } \\
$L W$ & $E B W$ & \multicolumn{2}{c}{ Daily net requirements $(g / k g E B W G)$} \\
\cline { 3 - 7 }$(\mathrm{kg})$ & $(\mathrm{kg})$ & $\mathrm{Ca}$ & $\mathrm{P}$ & $\mathrm{Mg}$ & $\mathrm{Na}$ & $\mathrm{K}$ \\
\hline 150 & 116 & 10,04 & 9,33 & 0,50 & 1,22 & 2,24 \\
200 & 163 & 10,64 & 9,36 & 0,46 & 1,18 & 2,37 \\
250 & 210 & 11,11 & 9,37 & 0,44 & 1,14 & 2,47 \\
300 & 257 & 11,49 & 9,38 & 0,42 & 1,12 & 2,56 \\
350 & 304 & 11,83 & 9,39 & 0,40 & 1,10 & 2,63 \\
400 & 352 & 12,12 & 9,40 & 0,39 & 1,08 & 2,69 \\
450 & 399 & 12,38 & 9,41 & 0,38 & 1,07 & 2,75 \\
\hline
\end{tabular}

de ganho de peso de corpo vazio (GPCVZ), valor próximo ao proposto pelo AFRC (1991), de 12,7 g/kg GPCVZ, e aos valores obtidos por PIRES et al. (1993a), de 12,84 g/kg GPCVZ, para bovinos Nelore não-castrados, SOARES (1994), de $12,10 \mathrm{~g} / \mathrm{kg}$ GPCVZ, para bovinos inteiros mestiços HolandêsNelore, bimestiços Fleckvieh-Angus x Nelore e búfalos, e PAULINO et al. (1999), de 10,87 g/kg GPCVZ, para animais de raças zebuínas, não-castrados. No entanto, foi maior que a exigência líquida para ganho observada por LANA et al. (1992), de $8,65 \mathrm{~g} / \mathrm{kg}$ GPCVZ, e inferior às propostas pelo ARC (1980), de 14,0 g/kg GPCVZ; por ARAÚJO et al. (1998), de 15,72 $\mathrm{g} / \mathrm{kg}$ GPCVZ; e por SIGNORETTI et al. (1998), de 16,96 g/kg GPCVZ. Para animais com $350 \mathrm{~kg}$ de PV, a exigência líquida de $\mathrm{Ca}$, foi estimada em $11,83 \mathrm{~g} / \mathrm{kg}$ GPCVZ, valor próximo ao observado por FERREIRA et al. (1999), de 10,86 g/kg GPCVZ, para novilhos F1 Simental-Nelore.

A conversão das exigências para ganho de $1 \mathrm{~kg}$ de GPCVZ, em exigências para ganho de $1 \mathrm{~kg}$ de ganho de peso vivo (GPV), foi feita utilizando o fator de correção de 1,19. O requerimento líquido de $P$ para animais de $300 \mathrm{~kg}$ estimado no presente trabalho, de $7,88 \mathrm{~g} / \mathrm{kg}$ GPV, é próximo ao relatado por PIRES et al. (1993a), de 7,90 g/kg de GPV, porém é menor que os requerimentos recomendados pelo NRC (1996), de 9,0 g/kg de GPV, e dos valores obtidos por ARAÚJO et al. (1998), de $8,77 \mathrm{~g} / \mathrm{kg}$ de GPV, e SIGNORETTI et al. (1999), de 8,27 g/kg de GPV. Por outro lado, foi maior que o requerimento recomendado pelo AFRC (1991), de 7,2 g/kg de GPV, e 
que os requerimentos relatados por SOARES (1994), de 5,73 g/kg de GPV; LANA et al. (1992), de 6,09 g/kg GPV; e PAULINO et al. (1999), de 5,25 g/kg GPV. Para animais com $350 \mathrm{~kg}$ de PV, a exigência líquida de $\mathrm{P}$, foi estimada em $7,89 \mathrm{~g} / \mathrm{kg} \mathrm{GPV}$, valor maior que o observado por FERREIRA et al. (1999), de $5,12 \mathrm{~g} / \mathrm{kg}$ de GPV, para novilhos F1 Simental-Nelore, devendo-se considerar que estes autores utilizaram como fator de correção o valor de 0,99 , para conversão das exigências para ganho de $1 \mathrm{~kg}$ de GPCVZ em exigências para ganho de $1 \mathrm{~kg}$ GPV.

Para $\mathrm{Mg}$, os requerimentos estimados neste trabalho, para animais com $300 \mathrm{~kg}$ de peso vivo, foram de $0,42 \mathrm{~g} / \mathrm{kg}$ GPCVZ e os estimados pelo ARC (1980), de 0,45 g/kg GPV; estes valores mostraram-se superiores aos propostos por LANA et al. (1992), de 0,26 g/kg GPCVZ; SOARES (1994), de 0,39 g/kg GPCVZ; PIRES et al. (1993b), de 0,35 g/kg GPCVZ; ARAÚJO et al. (1998), de 0,34 g/kg GPCVZ; SIGNORETTI et al. (1999), de 0,37 g/kg GPCVZ e PAULINO et al. (1999), de 0,25 g/kg GPCVZ. Para animais com $350 \mathrm{~kg}$ de $\mathrm{PV}$, a exigência líquida de $\mathrm{Mg}$ foi estimada em $0,40 \mathrm{~g} / \mathrm{kg} \mathrm{GPCVZ}$, superior à determinada por FERREIRA et al. (1999), de $0,31 \mathrm{~g} / \mathrm{kg}$ GPCVZ, para novilhos F1 Simental-Nelore.

Os requerimentos de Na para animais com $300 \mathrm{~kg}$ de peso vivo encontrados foram de $1,12 \mathrm{~g} / \mathrm{kg}$ GPCVZ. O ARC (1980) estimou valores de 1,50 g/kg GPV, enquanto outros autores sugeriram valores de $0,73 \mathrm{~g} / \mathrm{kg}$ GPCVZ (LANA et al., 1992); 0,89 g/kg GPCVZ (SOARES, 1994); 0,85 g/kg GPCVZ (PIRES et al., 1993b); 0,89 g/kg GPCVZ (ARAÚJO et al., 1998); 1,20 g/kg GPCVZ (SIGNORETTI et al., 1999); e $1,11 \mathrm{~g} / \mathrm{kg}$ GPCVZ (PAULINO et al., 1999). Para animais com $350 \mathrm{~kg}$ de $\mathrm{PV}$, a exigência líquida de $\mathrm{Na}$ foi estimada em $1,10 \mathrm{~g} / \mathrm{kg}$ GPCVZ, próxima à estimada por FERREIRA et al. (1999), de 1,32 g/kg GPCVZ, para novilhos F1 Simental-Nelore.

Do mesmo modo que os requerimentos de $\mathrm{Na}$, os requerimentos de $\mathrm{K}$ não apresentaram grandes variações em relação aos valores citados por outros autores, para animais com $300 \mathrm{~kg}$ de peso vivo. O requerimento de $\mathrm{K}$ para GPCVZ observado neste experimento foi de $2,56 \mathrm{~g} / \mathrm{kg} \mathrm{GPCVZ}$, enquanto o ARC (1980) sugere exigência de 2,0 g/kg GPV. Outros autores obtiveram valores de 1,36 g/kg GPCVZ (LANA et al., 1992), 1,96 g/kg GPCVZ (PIRES et al., 1993b); 1,65 g/kg GPCVZ (SOARES, 1994); 2,08 g/ kg GPCVZ (ARAÚJO et al., 1998), 1,88 g/kg GPCVZ (SIGNORETTI et al., 1999) e 1,67 g/kg GPCVZ (PAULINO et al., 1999). Para animais com $350 \mathrm{~kg}$ de $\mathrm{PV}$, a exigência líquida de $\mathrm{Na}$, foi estimada em $2,63 \mathrm{~g} / \mathrm{kg}$ GPCVZ, próxima à estimada por FERREIRA et al. (1999), de 1,52 g/kg GPCVZ, para novilhos F1 Simental-Nelore.

Na Tabela 7 encontram-se os requerimentos líquidos diários para mantença e os requerimentos dietéticos de $\mathrm{Ca}, \mathrm{P}, \mathrm{Mg}$, Na e K. Utilizando o fator de correção de 1,19 , para conversão das exigências para ganho de peso de corpo vazio em exigências para ganho de peso vivo, foram determinados os requerimentos dietéticos para ganho de $1 \mathrm{~kg}$ de peso vivo, adicionando-se a este valor os requerimentos dietéticos para mantença recomendados pelo AFRC (1991) e, dividindo-se o valor obtido pelos valores de biodisponibilidade de cada elemento propostos pelo ARC (1980) e AFRC (1991) (Tabela 2), determinou-se o requerimento dietético dos macrominerais estudados.

Tabela 7 - Requerimentos líquidos de mantença e requerimentos dietéticos diários de $\mathrm{Ca}, \mathrm{P}, \mathrm{Mg}, \mathrm{Na}$ e K (g/animal.dia), para novilhos mestiços dos tratamentos ganho contínuo e ganho compensatório

Table 7 - Net requirements of maintenance and daily dietary requirements of $\mathrm{Ca}, \mathrm{P}, \mathrm{Mg}, \mathrm{Na}$, and $\mathrm{K}$ (g/animal.day) for crossbred steers in continuous gain and compensatory gain groups ${ }^{1}$

\begin{tabular}{|c|c|c|c|c|c|c|c|c|c|c|}
\hline \multirow[t]{2}{*}{$\begin{array}{l}\text { PV } \\
L W\end{array}$} & \multicolumn{5}{|c|}{$\begin{array}{l}\text { Requerimento líquido de mantença } \\
\text { Net requirement of maintenance }\end{array}$} & \multicolumn{5}{|c|}{$\begin{array}{l}\text { Requerimento dietético } \\
\text { Dietary requirement }\end{array}$} \\
\hline & $\mathrm{Ca}$ & $\mathrm{P}$ & $\mathrm{Mg}$ & $\mathrm{Na}$ & $\mathrm{K}$ & $\mathrm{Ca}$ & $\mathrm{P}$ & $\mathrm{Mg}$ & $\mathrm{Na}$ & $\mathrm{K}$ \\
\hline 150 & 2,36 & 1,80 & 0,45 & 1,02 & 16,35 & 15,88 & 16,62 & 5,12 & 2,25 & 18,23 \\
\hline 200 & 3,14 & 2,40 & 0,60 & 1,36 & 21,44 & 17,77 & 17,70 & 5,80 & 2,58 & 23,43 \\
\hline 250 & 3,92 & 3,00 & 0,75 & 1,70 & 26,53 & 19,49 & 18,75 & 6,59 & 2,92 & 28,61 \\
\hline 300 & 4,71 & 3,60 & 0,90 & 2,04 & 31,61 & 21,13 & 19,80 & 7,37 & 3,28 & 33,76 \\
\hline 350 & 5,50 & 4,20 & 1,05 & 2,38 & 36,69 & 22,71 & 20,85 & 8,15 & 3,63 & 38,90 \\
\hline 400 & 6,28 & 4,80 & 1,20 & 2,72 & 41,78 & 24,21 & 21,90 & 8,99 & 3,99 & 44,04 \\
\hline 450 & 7,06 & 5,40 & 1,35 & 3,06 & 46,87 & 25,68 & 22,94 & 9,82 & 4,35 & 49,18 \\
\hline
\end{tabular}

1 Perdas endógenas, segundo o AFRC (1991)

${ }^{1}$ Endogenous losses suggested by AFRC (1991). 
O requerimento dietético diário de Ca preconizado pelo NRC (1996) para bovinos de corte em crescimento, com $300 \mathrm{~kg}$ de peso vivo e ganhando $1 \mathrm{~kg}$ por dia, é de $32,0 \mathrm{~g}$, estimado mediante o uso de coeficientes de absorção de $68 \%$. O requerimento diário de cálcio encontrado no presente trabalho, para animais de mesmo peso, foi de $21,13 \mathrm{~g}$, valor próximo ao proposto pelo AFRC (1991), de 18,71 g, e aos obtidos por LANA et al. (1992), de 18,60 g; PIRES et al. (1993), de 24,34 g; SOARES (1994), de 23,69 g; ARAÚJO et al. (1998), de 29,91 g; SIGNORETTI et al. (1999), de 26,30 g; e PAULINO et al. (1999), de 24,19 g. Para animais com $350 \mathrm{~kg}$ de PV e ganhando $1 \mathrm{~kg}$ por dia, o requerimento dietético diário de Ca foi de $22,71 \mathrm{~g}$, valor próximo ao obtido por FERREIRA et al. (1999), de 21,94 g, para novilhos F1 Simental-Nelore.

$\mathrm{O}$ requerimento dietético diário de $\mathrm{P}$ obtido para animais com $300 \mathrm{~kg}$ de PV e ganhando $1 \mathrm{~kg}$ por dia foi de $19,80 \mathrm{~g}$, valor próximo ao preconizado pelo NRC (1996), de 16,0 g, e aos obtidos por LANA et al. (1992), de 16,71 g; PIRES et al. (1993b), de 20,66 g; SOARES (1994), de 22,34 g; ARAÚJO et al. (1998), de 16,39 g; SIGNORETTI et al. (1999), de 17,40 g; e PAULINO et al. (1999), de 22,66 g, quando as perdas endógenas foram calculadas segundo o AFRC (1991). Entretanto, o requerimento de P encontrado neste trabalho foi superior ao preconizado pelo AFRC (1991), de 11,6 g diários. Para animais com $350 \mathrm{~kg}$ de PV e ganhando $1 \mathrm{~kg}$ por dia, o requerimento dietético diário de $\mathrm{P}$ foi de $20,85 \mathrm{~g}$, superior ao obtido por FERREIRA et al. (1999), de 7,53 g, para novilhos F1 Simental-Nelore.

Quanto ao requerimento dietético de $\mathrm{Mg}$, o ARC (1980) e o AFRC (1991) sugerem um valor de 1,35 g/kg GPCVZ, para animais com $300 \mathrm{~kg}$ de peso vivo, enquanto o NRC (1996) propõe requerimentos de $0,10 \%$ da MS da dieta, o que indica que bovinos de corte em crescimento requerem de 7,0 a 9,0 g de $\mathrm{Mg}$ por dia. $\mathrm{O}$ valor diário estimado neste trabalho para animais de mesmo peso foi de 7,37 g por animal por dia, o que está próximo às recomendações do NRC (1996) e aos valores obtidos por LANA et al. (1992), de 6,71 g; PIRES et al. (1993b), de 7,18 g; SOARES (1994), de 7,57 g; e PAULINO et al. (1999), de 6,65 g.

Os requerimentos dietéticos diários de sódio encontrados no presente experimento, para novilhos com $300 \mathrm{~kg}$ de peso vivo, foram de 3,28 g, valor próximo aos obtidos por LANA et al. (1992), de 2,98 g; PIRES et al. (1993b), de 3,08 g; SOARES (1994), de 3,06 g; e PAULINO et al. (1999), de 3,37 g, e superior aos valores recomendados pelo ARC (1980) e AFRC
(1991), de 1,50 g; por ARAÚJO et al. (1998), de 0,95 g; e por SIGNORETTI et al. (1999), de 1,55 g.

O requerimento de K relatado pelo ARC (1980) e AFRC (1991) é de 2,0 g, independente do peso vivo do animal, enquanto o NRC (1996) sugere que animais em confinamento requerem cerca de $0,6 \%$ da matéria seca consumida. Na literatura consultada, SOARES (1994) relatou requerimento dietético de K de 32,99 g, LANA et al. (1992), de 32,85 g, PIRES et al. (1993b), de 40,72 g e PAULINO et al. (1999), de $34,72 \mathrm{~g}$, valores próximos aos obtidos no presente experimento, de $33,76 \mathrm{~g}$, para animais com $300 \mathrm{~kg}$ de peso vivo.

\section{Conclusões}

O ganho de peso compensatório não afetou o conteúdo corporal nem as exigências de macroelementos minerais de novilhos mestiços Holandês-Gir.

As exigências líquidas de macroelementos minerais, para ganho de $1 \mathrm{~kg}$ de peso corporal vazio, de novilhos com peso entre 150 e $450 \mathrm{~kg}$, variaram de 10,04 a 12,38 g/kg GPCVZ para cálcio, 9,33 a 9,41 g/kg GPCVZ para fósforo, 0,50 a $0,38 \mathrm{~g} / \mathrm{kg} \mathrm{GPCVZ}$ para magnésio, 1,22 a 1,07 g/kg GPCVZ para sódio e 2,24 a $2,75 \mathrm{~g} / \mathrm{kg}$ GPCVZ para o potássio.

As exigências dietéticas de macroelementos minerais, para ganho de $1 \mathrm{~kg}$ de peso vivo, de novilhos com peso entre 150 e $450 \mathrm{~kg}$, variaram de 15,88 a 25,68 g para cálcio, 16,62 a 22,94 g para fósforo, 5,12 a 9,82 g para magnésio, 2,25 a 4,35 g para sódio e 18,23 a 49,18 g para o potássio.

\section{Referências Bibliográficas}

AGRICULTURAL AND FOOD RESEARCH COUNCIL AFRC. 1991. Technical Committee on Responses to Nutrients, Report Number 6. A Reappraisal of the Calcium and Phosphorous Requirements of Sheep and Cattle. Nutr. Abstr. Rev. (Series B), 61(9):576-612.

AGRICULTURAL RESEARCH COUNCIL - ARC. 1980. The nutrient requirements of farm livestock. London: Commonwealth Agricultural Bureaux. 351p.

ALMEIDA, M.I.V., FONTES, C.A.A., ALMEIDA, F.Q. et al. 2001. Conteúdo corporal e exigências líquidas de energia e proteína de novilhos mestiços Holandês-Gir em ganho compensatório. Rev. bras. zootec., 30(1):205-214.

AMMERMAN, C.B., GOODRICH, R.D. 1983. Advances in mineral nutrition in ruminants. J. Anim. Sci., 57(Suppl. 2):519.

ARAÚJO, G.G.L., COELHO DA SILVA, J.F., VALADARES FILHO, S.C. et al. 1998. Composição corporal e exigências líquidas e dietéticas de macroelementos minerais de bezerros alimentados com dietas contendo diferentes níveis de volumoso. R. Bras. Zootec., 27(5):1023-1030. 
CARVALHO, D.R. Composição corporal e exigências nutricionais de macroelementos inorgânicos de bovinos. Viçosa, MG: UFV, 1989. 84p. Dissertação (Mestrado em Zootecnia) - Universidade Federal de Viçosa, 1989.

CASTRO, A.C.G., COELHO DA SILVA, J.F., VALADARES FILHO, S.C. 1993. Composição corporal e exigências nutricionais de macroelementos inorgânicos para bovinos. $R$. Soc. Bras. Zootec., 22(2):360-371.

COELHO DA SILVA, J.F. Exigências de macroelementos inorgânicos para bovinos: o sistema ARC/AFRC e a experiência no Brasil. In: SIMPÓSIO INTERNACIONAL SOBRE EXIGÊNCIAS NUTRICIONAIS DE RUMINANTES, 1995, Viçosa, MG. Anais... Viçosa: UFV, 1995. p.467-504.

FERREIRA, M.A., VALADARES FILHO, S.C., MUNIZ, E.B. et al. 1999. Composição corporal e exigências líquidas de macroelementos minerais de bovinos $\mathrm{F}_{1}$ Simental x Nelore. Rev. bras. zootec., 28(2):361-367.

FONTES, C.A.A. 1995. Composição corporal, exigências líquidas de nutrientes para ganho de peso e desempenho produtivo de animais zebuínos e mestiços europeu-zebu. Resultados experimentais. In: SIMPÓSIO INTERNACIONAL SOBRE EXIGÊNCIAS NUTRICIONAIS DE RUMINANTES, 1995, Viçosa. Anais... Viçosa: UFV, 1995. p.419-455.

GRACE, N.D. 1983. Amounts and distribution of mineral elements associated with fleece-free body weight gains in grazing sheep. New Zeal. J. Agric. Res., 26:59-70.

GRAYBILL, F.A. 1976. Theory and application of the linear model. Massachussetts: Duxburg Press. 704p.

HANKINS, O.G., HOWE, P.E. 1946. Estimation of the composition of beef carcasses and cuts. S.e., USDA. (Technical Bulletin, 926).

HARVEY, W.R. 1987. Mixed model least squares and maximum likelihood computer program (LSMLWM) Versão PC - 1.

LANA, R.P., FONTES, C.C.A., PERON, A.J. et al. 1992. Composição corporal e do ganho de peso e exigências de energia, proteína e macroelementos minerais $(\mathrm{Ca}, \mathrm{P}, \mathrm{Mg}, \mathrm{Na}$ e K), de novilhos de cinco grupos raciais. 3. Conteúdo corporal e do ganho de peso e exigências de macroelementos minerais. R. Soc. Bras. Zootec., 21(3):538-544.

NATIONAL RESEARCH COUNCIL - NRC. 1984. Nutrient requirements of beef cattle. 6.ed. Washington, D.C. 90p.
NATIONAL RESEARCH COUNCIL - NRC. 1996. Nutrient requirements of beef cattle. 7.ed. Washington, D.C. 242p.

NOUR, A.Y.M., THONEY, M.L. 1988. Minerals of carcass soft tissue and bone of serially slaughtered cattle as affected by biological type and management. J. Agric. Sci., 111(1):41-49.

PAULINO, M.P., FONTES, C.C.A., JORGE, A.M. et al. 1999. Composição corporal e exigências de macroelementos minerais $(\mathrm{Ca}, \mathrm{P}, \mathrm{Mg}, \mathrm{Na}$ e $\mathrm{K})$ de bovinos de quatro raças zebuínas. Rev. bras. zootec., 28(3):634-641.

PIRES, C.C., FONTES, C.A.A., GALVÃO, J.G. et al. 1993a. Exigências nutricionais de bovinos de corte em acabamento. III. Exigências de cálcio e fósforo para ganho de peso. R. Soc. Bras. Zootec., 22(1):133-143.

PIRES, C.C., FONTES, C.A.A., GALVÃO, J.G. et al. 1993b. Exigências nutricionais de bovinos de corte em acabamento. IV. Exigências de magnésio, sódio e potássio. R. Soc. Bras. Zootec., 22(1):144-154.

ROBELIN, J., GEAY, Y. 1984. Body composition of cattle as affected by physiological status, breed, sex, and diet. In: GILCHRIST, F.M.C., MACKIE, R.I. (Eds.) Herbivore nutrition in the subtropics and tropics. Johannesburg: The Science Press. p.525-548.

SIGNORETTI, R.D., COELHO DA SILVA, J.F., VALADARES FILHO, S.C. et al. 1999. Composição corporal e exigências líquidas e dietéticas de macroelementos inorgânicos $(\mathrm{Ca}, \mathrm{P}$, $\mathrm{Mg}, \mathrm{K}$ e Na) de bezerros da raça Holandesa alimentados com dietas contendo diferentes níveis de volumoso. Rev. bras. zootec., 28(1):205-213.

SILVA, D.J. 1990. Análise de alimentos: métodos químicos e biológicos. Viçosa: UFV. 165p.

SOARES, J.E. Composição corporal e exigências de macroelementos minerais ( $\mathrm{Ca}, \mathrm{P}, \mathrm{Mg}$, Na e K) para ganho de peso em bovinos (zebuínos e taurinos) e bubalinos. Viçosa, MG: UFV, 1994. 77p. Dissertação (Mestrado em Zootecnia) - Universidade Federal de Viçosa, 1994.

Recebido em: 19/04/00 Aceito em:11/01/01 